\title{
Liver-specific reconstitution of CEACAM1 reverses the metabolic abnormalities caused by its global deletion in male mice
}

\author{
Lucia Russo $^{1}$ • Harrison T. Muturi ${ }^{1,2} \cdot$ Hilda E. Ghadieh ${ }^{1} \cdot$ Simona S. Ghanem $^{1}$ • \\ Thomas A. Bowman ${ }^{1} \cdot$ Hye Lim Noh ${ }^{3} \cdot$ Sezin Dagdeviren ${ }^{3} \cdot$ Godwin Y. Dogbey ${ }^{2}$. \\ Jason K. Kim ${ }^{3}$ - Garrett Heinrich ${ }^{2,4}$ • Sonia M. Najjar ${ }^{1,2,4}$
}

Received: 18 February 2017 / Accepted: 17 July 2017 /Published online: 14 September 2017

(C) Springer-Verlag GmbH Germany 2017

\begin{abstract}
Aims/hypothesis The carcinoembryonic antigen-related cell adhesion molecule 1 (CEACAM1) promotes insulin clearance. Mice with global null mutation $\left(\mathrm{Ccl}^{-/}\right)$or with liverspecific inactivation (L-SACC1) of $C c 1$ (also known as Ceacam1) gene display hyperinsulinaemia resulting from impaired insulin clearance, insulin resistance, steatohepatitis and obesity. Because increased lipolysis contributes to the metabolic phenotype caused by transgenic inactivation of CEACAM1 in the liver, we aimed to further investigate the primary role of hepatic CEACAM1-dependent insulin clearance in insulin and lipid homeostasis. To this end, we examined whether transgenic reconstitution of CEACAM1 in the liver of global $\mathrm{CCl}^{-/}$mutant mice reverses their abnormal metabolic phenotype.
\end{abstract}

Lucia Russo, Harrison T. Muturi and Hilda E. Ghadieh contributed equally to this study.

Electronic supplementary material The online version of this article (https://doi.org/10.1007/s00125-017-4432-y) contains peer-reviewed but unedited supplementary material, which is available to authorised users.

Sonia M. Najjar

najjar@ohio.edu

1 Center for Diabetes and Endocrine Research, College of Medicine and Life Sciences, University of Toledo, Toledo, OH, USA

2 Department of Biomedical Sciences, Heritage College of Osteopathic Medicine, Ohio University, Irvine Hall 229, 1 Ohio University, Athens, OH 45701-2979, USA

3 Division of Endocrinology, Metabolism and Diabetes, University of Massachusetts Medical School, Worcester, MA, USA

4 Diabetes Institute, Heritage College of Osteopathic Medicine, Ohio University, Athens, OH, USA
Methods Insulin response was assessed by hyperinsulinaemiceuglycaemic clamp analysis and energy balance was analysed by indirect calorimetry. Mice were overnight-fasted and refed for $7 \mathrm{~h}$ to assess fatty acid synthase activity in the liver and the hypothalamus in response to insulin release during refeeding.

Results Liver-based rescuing of CEACAM1 restored insulin clearance, plasma insulin level, insulin sensitivity and steatohepatitis caused by global deletion of $C c 1$. It also reversed the gain in body weight and total fat mass observed with $C c 1$ deletion, in parallel to normalising energy balance. Mechanistically, reversal of hyperphagia appeared to result from reducing fatty acid synthase activity and restoring insulin signalling in the hypothalamus.

Conclusions/interpretation Despite the potential confounding effects of deleting $\mathrm{Ccl}$ from extrahepatic tissues, liver-based rescuing of CEACAM1 resulted in full normalisation of the metabolic phenotype, underscoring the key role that CEACAM1-dependent hepatic insulin clearance pathways play in regulating systemic insulin sensitivity, lipid homeostasis and energy balance.

Keywords CEACAM1 · Energy balance $\cdot$ Fatty acid synthase · Hyperinsulinaemia · Insulin clearance $\cdot$ Insulin resistance $\cdot$ Lipolysis $\cdot$ Normoinsulinaemia $\cdot$ Steatohepatitis

$\begin{array}{ll}\text { Abbreviations } \\ \text { ApoA-1 } & \begin{array}{l}\text { Apolipoprotein A-1 } \\ \text { BAT }\end{array} \\ \text { CEACAMn } 1 & \begin{array}{l}\text { Carcinoembryonic antigen-related cell } \\ \text { adhesion molecule } 1\end{array} \\ \text { ECL } & \text { Enhanced chemiluminescence } \\ \text { FAO } & \text { Fatty acid } \beta \text {-oxidation } \\ \text { FASN } & \text { Fatty acid synthase }\end{array}$


L-CC1 Transgenic mice with liver-specific overexpression of wild-type rat $C c 1$ driven by ApoA1 promoter

L-SACC1 Transgenic mice with liver-specific overexpression of the dominant-negative serine to alanine phosphorylation-defective mutant of rat CEACAM1

$\operatorname{IR} \alpha \quad \alpha$ subunit of the insulin receptor

$\operatorname{IR} \beta \quad \quad \beta$ subunit of the insulin receptor

$\mathrm{m} C c \mathrm{P} \quad$ Mouse $C c 1$

PPAR $\alpha \quad$ Peroxisome proliferator-activated receptor- $\alpha$

$\mathrm{rCcl} \quad$ Rat $C c l$

SREBP-1c Sterol regulatory element binding protein-1c

WAT White adipose tissue

\section{Introduction}

Obesity and type 2 diabetes are characterised by insulin resistance, hyperinsulinaemia and altered lipid and glucose homeostasis [1]. Whereas hyperinsulinaemia can result from increased insulin secretion to compensate for insulin resistance, it can also emerge when insulin clearance is impaired, causing insulin resistance, at least in part, by downregulating insulin receptors [2, 3]. Moreover, chronic hyperinsulinaemia limits the pulsatility of insulin release [4], restricting insulinmediated activation of insulin receptor and downstream signalling pathways in hepatocytes.

Endogenous insulin is mostly cleared in the liver. Consistent with the predominant expression of the carcinoembryonic antigen-related cell adhesion molecule 1 (CEACAM1) in the liver [5], we have shown that CEACAM1 provides a postreceptor mechanistic underpinning of how impaired insulin clearance causes chronic hyperinsulinaemia [6-8]. Upon its phosphorylation by the insulin receptor [9], CEACAM1, a plasma membrane glycoprotein, increases the rate of receptormediated insulin uptake into the hepatocyte and its targeting to the degradation pathways by taking part in the insulin receptor endocytosis complex [10]. The regulatory effect of CEACAM1 in insulin clearance is bolstered by impaired insulin clearance followed by chronic hyperinsulinaemia in transgenic mice with liver-specific overexpression of the dominant-negative serine to alanine phosphorylation-defective mutant of rat CEACAM1 (L-SACC1) or with global deletion of the $C c 1$ (also known as Ceacam 1$)$ gene $\left(\mathrm{CCl}^{-/}\right)$. Like L-SACC1 mice [6], $\mathrm{Ccl}^{-/-}$mice manifest impaired insulin clearance and hyperinsulinaemia at 2 months of age [7]. While they simultaneously develop insulin resistance when the mutation is propagated on the mixed C57BL/6x129sv genetic background, they do not develop systemic insulin resistance until 5-6 months of age when the mutation is propagated on $\mathrm{C} 57 \mathrm{BL} / 6$, as demonstrated by hyperinsulinaemic-euglycaemic clamp analysis [7]. Intact beta cell mass and glucose-stimulated insulin secretion and fasting normoglycaemia in $\mathrm{Ccl}^{-/-}$and L-SACC1 mice demonstrate that their hyperinsulinaemia is primarily caused by impaired insulin clearance [7].

Hyperinsulinaemia in $\mathrm{Cc}^{-/-}$mutants also induces hepatic lipid production followed by redistribution to white adipose tissue (WAT) to contribute to visceral obesity [7, 11, 12]. Loss of the repressive CEACAM1-mediated acute negative effect of insulin on fatty acid synthase (FASN) activity under hyperinsulinaemic conditions [13] probably contributes to increased de novo lipogenesis and hepatic steatosis in these mice.

Extrahepatic factors affect insulin action and fat accumulation in the liver of $C_{c} 1^{-/-}$mice. For example, inhibiting lipolysis and inducing fatty acid $\beta$-oxidation by L-carnitine restores insulin clearance and insulin action in L-SACC1 transgenic mice [14]. This suggests that altered fat metabolism plays an important role in sustained insulin resistance. In fact, by inducing human Apolipoprotein A-1 (ApoA-1) [15] and activating the peroxisome proliferation activated receptor $(\operatorname{PPAR} \alpha)$ that represses $C c 1$ expression [16], elevated release of NEFAs from adipose tissue maintains ApoA-1-driven expression of the phosphorylation-defective rat S503A CEACAM1 mutant at a higher level than that of the mouse endogenous $C c l$ gene, thus, contributing to the dominantnegative effect of the rat $C c 1$ transgene on insulin clearance in L-SACC1 mice $[14,17]$. Whereas this points to the important role of an extrahepatic factor (lipolysis) in the pathogenesis of insulin resistance in L-SACC1 mice, it does not fully explore the primary role of hepatic CEACAM1-dependent insulin clearance pathways in regulating insulin homeostasis and action. Therefore, we rescued CEACAM1 expression exclusively in the livers of $\mathrm{CCl}^{-1-}$ mice and investigated whether it reverses altered insulin and fat metabolism.

\section{Methods}

Mice generation To obtain mice heterozygous for the $C c 1$ locus, C57BL/6J.Ccl ${ }^{-1-}$ mice $[7,12]$ were crossed with transgenic mice with liver-specific overexpression of wild-type rat Cc1 driven by ApoA-1 promoter (L-CC1) [15]. These were backcrossed with C57BL/6J mice (Jackson Laboratory, Bar Harbor, ME, USA) six times to obtain a progeny of $\mathrm{CCl}^{+/-}$ with or without the transgene. Intercrossing this progeny produced several genotypes including $\mathrm{CCl}^{-/-}$with the transgene $\left(\mathrm{Ccl}^{- \text {-xliver+ }}\right)$ and control littermates: $\mathrm{Ccl}^{-/-}$without the transgene $\left(\mathrm{Ccl}^{-/-}\right), \mathrm{Ccl}^{+/+}$without the transgene $\left(\mathrm{Ccl}^{+/+}\right)$ and $\mathrm{CCl}^{+/+}$with the transgene (L-CC1). Genotypes were identified by PCR-based genotyping using ear genomic DNA and primers for the wild-type allele (exon 2), the knockout allele (the neomycin cassette between exon 7 and 9) and the rat transgene (exons 6 and 9) (see ESM Fig. 1). 
Male mice were kept in a $12 \mathrm{~h}$ light/dark cycle under pathogen-free conditions and fed a standard diet (Harlan Laboratories, Teklad 2016, Haslett, MI, USA) ad libitum [15]. In some experiments, mice were injected i.p. once daily with nicotinic acid (200 mg/kg body weight per day; SigmaAldrich, Saint Louis, MO, USA) for 2 days [17]. Mice were placed in cages with Alpha-dri bedding (Shepherd Specialty Papers, Cincinnati, OH, USA) prior to food removal and phenotyping. The Institutional Animal Care and Utilization Committee approved all procedures. Animals and samples were not randomised. No data, samples or animals were excluded in analyses.

Body composition Whole body composition was assessed in awake mice by NMR technology (Bruker Minispec, Billerica, MA, USA). Magnetic resonance spectroscopy $\left({ }^{1} \mathrm{H}-\mathrm{MRS}\right.$; Echo Medical Systems, Houston, TX, USA) was used to determine fat and lean mass as percentage of total body weight, as previously described [15].

Metabolic variables Retro-orbital venous blood was drawn at 11:00 hours from mice fasted overnight to measure plasma insulin (80-INSMSU-E01 ELISA kit; ALPCO, Salem, NH, USA), C-peptide (80-CPTMS-E01 ELISA kit; ALPCO), leptin (22-LEPHUU-E01 ELISA kit; ALPCO), NEFA (NEFA-C enzymatic colorimetric assay; Wako, Richmond, VA, USA), and plasma and hepatic triacylglycerol levels (Pointe Scientific, enzymatic colorimetric assays, Canton, MI, USA) [7, 12, 18].

Hyperinsulinaemic-euglycaemic clamp analysis A $2 \mathrm{~h}$ hyperinsulinaemic-euglycaemic clamp was performed in awake overnight-fasted mice with primed and continuous infusion of human soluble insulin (Humulin, Lilly, Indianapolis, IN, USA) at a rate of $2.5 \mathrm{mU} \mathrm{kg}^{-1} \mathrm{~min}^{-1}$ [7]. Glucose metabolism was estimated with a continuous infusion of $1850 \mathrm{~Bq}$ of $\left[3-{ }^{3} \mathrm{H}\right]$ glucose (Perkin-Elmer, Shelton, CT, USA) and then $3700 \mathrm{~Bq}$ throughout the clamp.

C75 treatment and daily food intake Food was removed from individually housed mice at 15:00 hours and weighed for 4 days. At 18:00 hours, mice were injected i.p. with a daily dose of $30 \mathrm{mg} / \mathrm{kg}$ body weight of C75 (Calbiochem, EMD Bioscience, Bedford, MA, USA) dissolved in RPMI 1640 medium (Gibco, Gaithersburg, MD, USA). Food was returned 5 min before the dark cycle began at 19:00 hours and the weight of ingested food/day was calculated.

Biotin-labelling of primary hepatocytes Primary hepatocytes were isolated by perfusing livers with collagenase-type II $(1 \mathrm{mg} / \mathrm{ml})$ (Worthington, Lakewood, NJ, USA) and plated in six-well plates in complete Williams-E medium at $1 \times 10^{6}$ cells/well [6]. After $24 \mathrm{~h}$, cells were incubated in the absence or presence of $100 \mathrm{nmol} / \mathrm{l}$ insulin (Sigma-Aldrich) at $37^{\circ} \mathrm{C}$ for $5 \mathrm{~min}$, followed by incubation with biotin $(1 \mathrm{mg} / \mathrm{ml}$ ) (Pierce, Rockford, IL, USA) in PBS for $30 \mathrm{~min}$ on ice. Cells were lysed in $1 \%$ Triton- $\mathrm{X}$ and subjected ( $30 \mu \mathrm{g}$ proteins) to immunoprecipitation at $4{ }^{\circ} \mathrm{C}$ with streptavidin (Pierce). Immunoprecipitates were centrifuged and analysed by $7 \%$ SDS-PAGE and immunoblotting with 1:1000 of insulin receptor alpha $(\operatorname{IR} \alpha)$ antibody (N-20; Santa Cruz, Dallas, TX, USA) or a custom-made Ab3759 polyclonal antibody against purified mouse CEACAM1, as titrated [12, 19], followed by horseradish peroxidase-conjugated mouse anti-rabbit IgG antibody (Jackson Immunoresearch, West Grove, PA, USA) and subjected to enhanced chemiluminescence (ECL; Amersham Pharmacia, Sunnyvale, CA, USA).

FASN activity Livers were homogenised, centrifuged and the supernatant fraction was added to a reaction mix containing $3700 \mathrm{~Bq}\left[{ }^{14} \mathrm{C}\right]$ malonyl-CoA (Perkin-Elmer) and $25 \mathrm{nmol}$ malonyl-CoA in the absence or presence of $500 \mu \mathrm{mol} / 1$ NADPH (Sigma-Aldrich) [13]. The reaction was stopped with 1:1 chloroform:methanol solution and samples were centrifuged, butanol-extracted and counted. FASN activity was calculated as the cpm of $\left[{ }^{14} \mathrm{C}\right]$-incorporated $\mathrm{Bq} / \mu \mathrm{g}$ cell lysates.

Ex vivo palmitate oxidation The liver was extracted, homogenised and incubated in $0.2 \mathrm{mmol} / 1$ of $\left[1-{ }^{14} \mathrm{C}\right]$ palmitate $\left(185 \times 10^{5} \mathrm{~Bq}\right)$ (American Radiolabeled Chemicals, St Louis, MO, USA) and $2 \mathrm{mmol} / \mathrm{l}$ ATP [17]. The reaction was terminated with perchloric acid to recover radioactive acid soluble metabolites. Trapped $\mathrm{CO}_{2}$ was measured by liquid scintillation. The oxidation rate was expressed as the sum of total and partial fatty acid oxidation expressed in $\mathrm{nmol} \mathrm{g}{ }^{-1} \mathrm{~min}^{-1}$.

Western blot analysis This was achieved using 1:1000 polyclonal antibodies against phosphorylated insulin receptor beta (p-IR $\beta$; phospho-Y1361), IR $\beta$ (C18C4) (Abcam, Cambridge, MA, USA), phosphorylated Akt (p-Ser473 Akt), Akt, $\alpha$-FASN (Cell Signaling, Danvers, MA, USA), custommade rabbit polyclonal Ab2456 against the mouse CEACAM1 extracellular domain, as titrated [20], and custom-made rat CEACAM1 $(\alpha \mathrm{P} 3[\mathrm{Y} 488])$ and phosphorylated CEACAM1 ( $\alpha$-p-CEACAM1) mouse antibodies (Bethyl Laboratories, Montgomery, TX, USA), as titrated [16, 21]. $\alpha$ Actin, $\alpha$-GAPDH and $\alpha$-tubulin antibodies (Santa Cruz) were used at 1:5000 dilution for normalisation. Blots were incubated with horseradish peroxidase-conjugated donkey anti-rabbit $\mathrm{IgG}$ antibody, as per manufacturer's titration, for analysis of transfected, knockout or siRNA-knockdown cells (GE Healthcare Life Sciences, Amersham, Sunnyvale, CA, USA), followed by ECL. 
Quantitative real-time PCR cDNA was synthesised using iScript cDNA Synthesis Kit (Bio-Rad, Hercules, CA, USA) and qPCR was performed using Fast SYBR Green Master Mix by the ABI StepOnePlus Real-Time PCR System (Applied Biosystems, Foster City, CA, USA) [15] and $10 \mu \mathrm{mol} / 1$ primers for $C c 1$ (mouse $C c 1$ [ $\mathrm{mCcl}$ ], rat $C c 1$ [rCcl] and total Ccl), Tnfo (Tnf), F4/80 (Adgrel), Il-1 $\beta$ (Illb), Srebp-1c (Srebf1), Fasn, Fgf21, Cpt1 $\alpha$ (Cptla), Ppara (Ppara), Ucp1, Cox2 (ESM Table 1). mRNA was normalised to Gapdh, unless otherwise stated.

Statistical analysis Parametric data were analysed by oneway ANOVA with Tukey's test for multiple comparisons. Nonparametric data were analysed by the Kruskal-Wallis test with Dunn's correction for multiple pairwise comparisons. All data were expressed as means \pm SEM using GraphPad Prism 6 software (www.graphpad.com, downloaded on 29 June 2016). A $p$ value $\leq 0.05$ was considered statistically significant.

\section{Results}

Hepatic-specific rescuing of CEACAM1 qPCR analysis demonstrated that mouse mRNA $(\mathrm{mCcl})$ is expressed in the liver, hypothalamus and hepatocyte of $\mathrm{Ccl}^{+/+}$and L-CC1 mice, but not $\mathrm{Ccl}^{-1-}$ or $\mathrm{Ccl}^{-/- \text {xliver+ }}$ mice (Table 1). The rat mRNA ( $\mathrm{CCc}$ ) was detected in the liver and hepatocytes of L$\mathrm{CCl}$ and $\mathrm{Ccl}^{-/-x l i v e r+}$ mice, but not $\mathrm{Ccl}^{+/+}$and $\mathrm{Ccl}^{-/-}$mice.

Table 1 mRNA analysis of $C c 1$ expression

\begin{tabular}{|c|c|c|c|c|}
\hline & $\mathrm{Ccl}^{+/+}$ & $\mathrm{Ccl}^{-/-}$ & $\mathrm{L}-\mathrm{CC} 1$ & $C c 1^{-1-\text { xliver }+}$ \\
\hline \multicolumn{5}{|l|}{$\mathrm{mCcl} \mathrm{mRNA}$} \\
\hline Liver & $0.94 \pm 0.04$ & Negl. & $0.90 \pm 0.03$ & Negl. \\
\hline Hepatocytes & $3.03 \pm 0.56$ & Negl. & $2.65 \pm 0.62$ & Negl. \\
\hline Hypothalamus & $1.27 \pm 0.04$ & Negl. & $1.20 \pm 0.04$ & Negl. \\
\hline WAT & Negl. & Negl. & Negl. & Negl. \\
\hline \multicolumn{5}{|l|}{$\mathrm{rCcl}$ mRNA } \\
\hline Liver & Negl. & Negl. & $5.09 \pm 1.06$ & $6.75 \pm 1.26$ \\
\hline Hepatocytes & Negl. & Negl. & $4.34 \pm 0.39$ & $4.48 \pm 0.63$ \\
\hline Hypothalamus & Negl. & Negl. & Negl. & Negl. \\
\hline WAT & Negl. & Negl. & Negl. & Negl. \\
\hline \multicolumn{5}{|l|}{ Total $C c 1$ mRNA } \\
\hline Liver & $1.19 \pm 0.09$ & Negl. & $2.47 \pm 0.19$ & $1.18 \pm 0.08$ \\
\hline
\end{tabular}

Values are expressed as mean \pm SEM

RNA was analysed in triplicate in tissues or primary cells of male mice $(n=5 /$ genotype $)$

Gene expression was normalised to $18 \mathrm{~S}$

Total $C c 1$ mRNA $=\mathrm{r} C c 1+\mathrm{m} C c 1$ mRNA

Negl., negligible
Total rat and mouse mRNA levels were about twofold higher in the livers of L-CC1 mice compared with $\mathrm{Ccl}^{+/+}$and $\mathrm{Ccl}^{-/-x l i v e r+}$ mice (Table 1). $\mathrm{rCcl}$ levels were negligible in the hypothalamus of all genotypes. Neither $\mathrm{rCcl}$ nor $\mathrm{mCcl}$ were detected in the WAT of any genotype [5]. Nevertheless, CEACAM1 may still be targeted to an undetermined extrahepatic cell/tissue.

Western blot analysis using a polyclonal antibody against the extracellular domain of mouse CEACAM1 (IB: $\alpha$-mCC1) detected CEACAM1 in the livers of $\mathrm{Ccl}^{+/+}$and L-CC1 mice, but not $\mathrm{Ccl}^{-/ \text {xliver+ }}$ mice (Fig. 1a). Immunoblotting with rat $\alpha$-CEACAM1 (IB: $\alpha$-rCC1) revealed the rat protein in the livers of $\mathrm{L}-\mathrm{CCl}$ and $\mathrm{Ccl}^{-/-x l i v e r+}$ mice, but not $\mathrm{Ccl}^{+/+}$mice (Fig. 1a). Rat CEACAM1 was not expressed in other tissues in $\mathrm{Ccl}^{-/ \text {-xliver+ }}$ mice, including the small intestine [15], the other main site of ApoA-1 production, kidney and heart (Fig. 1a, b).

Hepatic CEACAM1 rescuing prevents impaired insulin clearance and insulin resistance in $\mathrm{Ccl}^{-1-}$ mice $\mathrm{Ccl}^{-/-}$mice exhibited higher body weight, and total and relative fat mass than $\mathrm{CcI}^{+/+}$mice from 2 months of age (Table 2). Moreover, they exhibited lower relative, but not total, lean mass (Table 2). Liver-specific rescuing of CEACAM1 reversed these abnormalities by 6 months of age (Table 2).

$\mathrm{Ccl}^{-/-}$mice also exhibited hyperinsulinaemia (Fig. 1c) resulting from impaired insulin clearance, as indicated by steady-state C-peptide/insulin molar ratio at 2 months of age (Fig. 1e) [7]. Immunoblotting the biotin-streptavidin immunopellet with polyclonal antibodies against $\operatorname{IR} \alpha$ and whole mouse CEACAM1 (Fig. 1f) revealed that insulininduced insulin receptor and CEACAM1 internalisation, measured by the loss of biotin-labelled surface membrane proteins in cells treated with insulin vs buffer [6], was almost completely blunted in hepatocytes from $\mathrm{Ccl}^{-1-}$ mice. Thus, receptor-mediated insulin internalisation (and degradation) is compromised in $\mathrm{Ccl}^{-/-}$hepatocytes.

Liver-specific rescuing of CEACAM1 prevented impairment of insulin clearance and hyperinsulinaemia (Fig. 1d, c). This appears to be due to the recovery of insulin-induced internalisation of the insulin-receptor-CEACAM1 complex, as shown by reduced insulin-mediated biotin-labelled IR $\alpha$ levels in $\mathrm{Ccl}^{-/-x \text { liver+ }}$ mice (Fig. 1f).

$\mathrm{Ccl}^{-/-}$mice exhibited fed but not fasting hyperglycaemia, which was prevented by liver-specific CEACAM1 rescuing (Table 2) [7]. To further assess the effect of hepatic CEACAM1 on insulin action in vivo, a $2 \mathrm{~h}$ hyperinsulinaemic-euglycaemic clamp analysis was performed on overnight-fasted, awake 6-month-old mice, since $\mathrm{Ccl}^{-/-}$mice develop insulin resistance at this age [7] (Fig. 2). Basal glucose at the beginning and during the clamp were similar in all groups (Fig. 2a). Whereas basal insulin was elevated in $\mathrm{Ccl}^{-/-}$mice, insulin levels were maintained at comparable levels in all groups (Fig. 

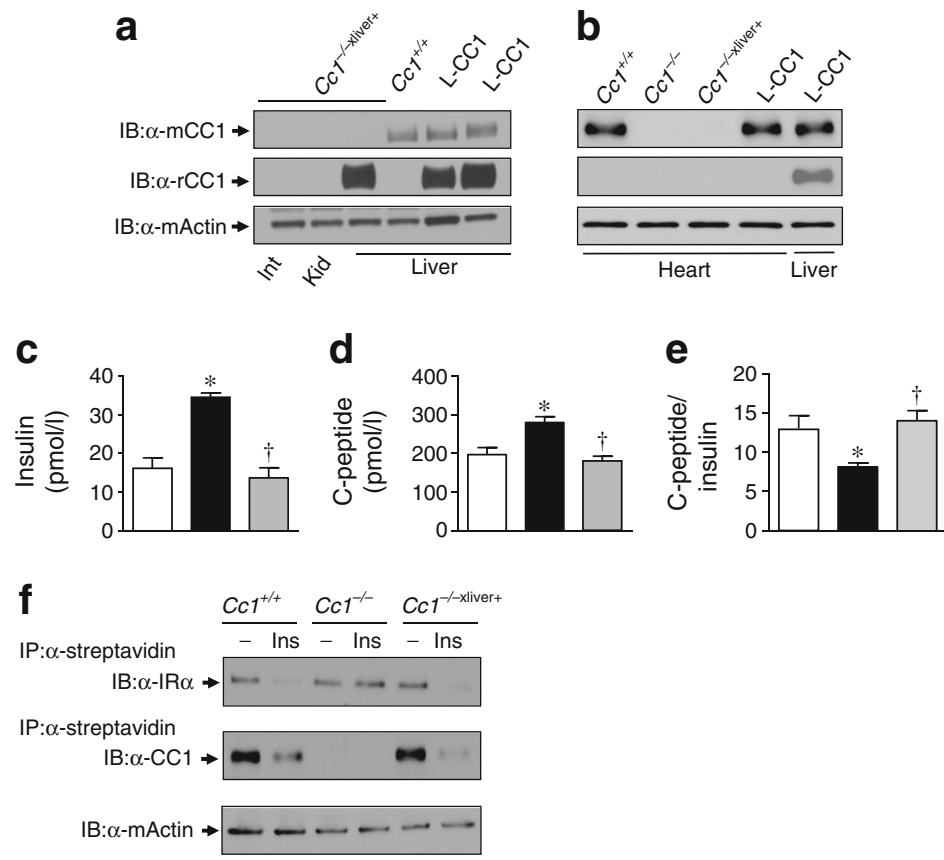

Fig. 1 Tissue-specific expression of the transgene. (a-b) Mouse (mCC1) and rat (rCC1) CEACAM1 protein content in intestine (Int), kidney (Kid), heart and liver were analysed by immunoblotting with polyclonal antibodies $(\alpha)$. Immunoblotting with $\alpha$-mActin was used to normalise for loading. (c-e) $C_{c} l^{+/+}$(white bars), $C c 1^{-/}$(black bars) and $C c 1^{-/ \text {xliver+ }}$ (grey bars) mice ( $n=5 /$ genotype; 2 months old) were fasted overnight and retro-orbital blood was drawn to assess plasma insulin (c) and C-peptide (d) levels to calculate steady-state C-peptide/insulin molar ratio (e) as a measure of insulin clearance. Assays were performed in triplicate. Values are expressed as mean $\pm \mathrm{SEM} ;{ }^{*} p \leq 0.05$ vs $C c l^{+/+},{ }^{\dagger} p \leq 0.05$ vs

$2 b)$. The glucose infusion rate required to maintain euglycaemia was reduced in the insulin-resistant $\mathrm{Ccl}^{-/}$mice (Fig. 2c) but normal in $\mathrm{Ccl}^{-/ \text {-xliver+ }}$ mice, emphasising the role of hepatic CEACAM1 in maintaining systemic insulin sensitivity. Whereas insulin-stimulated whole-body glycogen synthesis was low in $\mathrm{Ccl}^{--}$mice, it was normal in $\mathrm{Ccl}^{- \text {-xliver+ }}$ mice in comparison with $\mathrm{Ccl}^{+/+}$mice (Fig. 2d). Basal hepatic glucose production (Fig. 2e), a measurement of the appearance rate in pre-clamp conditions, was similar in all groups. In contrast to $\mathrm{Ccl}^{-/-}$mice, insulin comparably suppressed hepatic glucose production in $\mathrm{Ccl}^{-/ \text {xliver+ }}$ and $\mathrm{Ccl}^{+/+}$mice (Fig. 2e, f). Whereas whole body glucose turnover was reduced in $\mathrm{Ccl}^{-/-}$ mice, it was normal in $\mathrm{Ccl}^{-/ \text {xliver+ }}$ mice (Fig. 2g). Consistently, reduction in glucose uptake in the gastrocnemius muscle (Fig. 2h), WAT (Fig. 2i) and brown adipose tissue (BAT) (Fig. 2j) was also prevented in $\mathrm{Ccl}^{-/ \text {xliver+ }}$ mice. The data demonstrate that liver-specific rescuing of CEACAM1 curbed impaired hepatic insulin clearance and systemic insulin resistance caused by $C c 1$ deletion.

Hepatic CEACAM1 rescuing prevents fat accumulation and inflammation in $\mathrm{CcI}^{-/-}$livers $\mathrm{Ccl}^{-/-}$exhibited hepatic triacylglycerol accumulation compared with $\mathrm{CcI}^{+/+}$mice (Table 2). Histological evaluation of liver sections stained
$\mathrm{Ccl}^{-/-}$. (f) Primary hepatocytes of $\mathrm{Ccl}^{+/+}, \mathrm{Ccl}^{-/-}$and $\mathrm{Ccl}^{-/ \text {xliver+ }}$ mice were treated with buffer (-) or insulin (Ins) before cell-surface proteins were labelled with biotin. Proteins were immunoprecipitated with $\alpha$ streptavidin beads prior to analysis by $7 \%$ SDS-PAGE and immunoblotting with antibodies against IR $\alpha$ and whole mouse CEACAM1 with cross-reactivity with the rat protein $(\mathrm{CC} 1)$. Total lysates were also analysed by immunoblotting with $\alpha$-mActin. For (a), (b) and (f), gels represent more than two experiments (different mice per genotype per experiment). IB, immunoblotting; IP, immunoprecipitation

using $H \& E$ revealed diffuse fat infiltration in the livers of $\mathrm{Ccl}^{-/-}$mice, as opposed to $\mathrm{Ccl}^{+/+}$in which the parenchyma was fat-free (Fig. 3). The steatosis appeared predominantly microvesicular with a mix of macrosteatosis. Liver-specific rescuing of CEACAM1 reversed hepatic triacylglycerol level (Table 2) and fat deposition (Fig. 3d vs Fig. 3b, $\mathrm{Ccl}^{-/-x \text { liver+ }}$ vs $\left.\mathrm{Ccl}^{-/-}\right)$. This appears to result in part from elevated fatty acid $\beta$-oxidation (FAO), as supported by conversion to $\mathrm{CO}_{2}$ (Fig. 3e; $\mathrm{Ccl}^{-/- \text {xliver+ }}$ vs $\left.C c 1^{-/-}\right)$, and elevated mRNA levels of Fgf21 (Fig. 3f) and other genes involved in FAO (Ppara, Cpt $1 \alpha$ and Ucpl) (ESM Table 2). Reduced hepatic steatosis in $\mathrm{Ccl}^{-/-x \text { liver+ }}$ mice can also arise from the reversal of de novo lipogenesis in $\mathrm{Ccl}^{-/}$mice, as indicated by the lower basal Fasn mRNA levels in association with reversal of hyperinsulinaemia (Fig. $3 \mathrm{~g}, \mathrm{~h} ; \mathrm{Ccl}^{-/-x l i v e r+}$ vs $\mathrm{Ccl}^{-/-}$), likely mediated by sterol regulatory element binding protein-1c (SREBP-1c) inactivation (ESM Table 2) [22]. Moreover, liver-specific rescuing of CEACAM1 restored the suppressive effect of insulin on FASN activity in mice refed for $7 \mathrm{~h}$ after an overnight fast (Fig. 3k). As shown by Najjar et al [13], insulin downregulates FASN activity in normoinsulinaemic mice $\left(\mathrm{Ccl}^{+/+}, \mathrm{L}-\mathrm{CC} 1, \mathrm{Ccl}^{-/-x l i v e r+}\right)$ by activating the IR $\beta$ subunit (Fig. 3i, IB: $\alpha$-p-IR $\beta$ ), which causes CEACAM1 phosphorylation (Fig. 3j, IB: $\alpha$-p-CEACAM1) and its binding to FASN 
Table 2 Plasma and tissue biochemistry in male mice at 2-3 months and 5-6 months

\begin{tabular}{|c|c|c|c|c|c|c|c|}
\hline \multirow[t]{2}{*}{ Variable } & \multirow[t]{2}{*}{$n$} & \multicolumn{3}{|l|}{$2-3$ months } & \multicolumn{3}{|l|}{$5-6$ months } \\
\hline & & $\mathrm{CCl}^{+/+}$ & $\mathrm{Ccl}^{-/-}$ & $C c 1^{-/- \text {xliver+ }}$ & $\mathrm{CCl}^{+/+}$ & $\mathrm{CCl}^{-/-}$ & $C c 1^{-1-\text { xliver+ }}$ \\
\hline BW (g) & 4 & $21.3 \pm 0.5$ & $23.0 \pm 0.5^{*}$ & $18.3 \pm 0.4 *{ }^{*}$ & $25.7 \pm 0.7$ & $30.4 \pm 1.4^{*}$ & $26.0 \pm 1.1^{\dagger}$ \\
\hline$\%$ Fat mass/BW & 4 & $0.6 \pm 0.4$ & $3.9 \pm 0.4 *$ & $2.1 \pm 0.4^{*}, \dagger$ & $4.3 \pm 1.4$ & $13.0 \pm 2.8^{*}$ & $6.0 \pm 1.2^{\dagger}$ \\
\hline$\%$ Lean mass/BW & 4 & $73.1 \pm 0.5$ & $68.2 \pm 0.7^{*}$ & $70.0 \pm 0.3^{*}$ & $70.2 \pm 1.2$ & $62.8 \pm 2.2 *$ & $68.0 \pm 1.0^{\dagger}$ \\
\hline Total fat mass (g) & 4 & $0.1 \pm 0.1$ & $0.8 \pm 0.1 *$ & $0.5 \pm 0.1^{* \dagger}$ & $1.4 \pm 0.5$ & $4.5 \pm 1.1^{*}$ & $1.5 \pm 0.4^{\dagger}$ \\
\hline Total lean mass (g) & 4 & $16.0 \pm 0.4$ & $15.7 \pm 0.4$ & $12.9 \pm 0.3^{*, \dagger}$ & $20.9 \pm 0.5$ & $22.8 \pm 0.9$ & $21.5 \pm 0.8^{\dagger}$ \\
\hline BW MRS (g) & $8-9$ & na & na & na & $24.2 \pm 0.6$ & $26.6 \pm 0.8^{*}$ & $24.3 \pm 0.3^{\dagger}$ \\
\hline Fat mass MRS (g) & $8-9$ & na & na & na & $1.8 \pm 0.2$ & $4.7 \pm 0.5^{*}$ & $1.9 \pm 0.3^{\dagger}$ \\
\hline Visceral adiposity (\% BW) & 5 & $1.1 \pm 0.1$ & $1.4 \pm 0.2$ & $1.1 \pm 0.1$ & $1.6 \pm 0.2$ & $3.2 \pm 0.7 *$ & $1.4 \pm 0.2^{\dagger}$ \\
\hline Liver weight/BW (\%) & 5 & $3.9 \pm 0.2$ & $4.2 \pm 0.1$ & $4.2 \pm 0.1$ & $4.2 \pm 0.1$ & $4.3 \pm 0.3$ & $4.0 \pm 0.1$ \\
\hline Plasma NEFA (mmol/l) & 5 & $1.1 \pm 0.2$ & $1.5 \pm 0.2 *$ & $1.1 \pm 0.1^{\dagger}$ & $1.6 \pm 0.2$ & $2.9 \pm 0.6^{*}$ & $1.4 \pm 0.1^{\dagger}$ \\
\hline Plasma leptin $(\mu \mathrm{g} / \mathrm{l})$ & $7-8$ & na & na & na & $3.5 \pm 1.3$ & $18.6 \pm 0.8^{*}$ & $4.2 \pm 1.2^{\dagger}$ \\
\hline Plasma TG (mmol/l) & 10 & $81.1 \pm 3.8$ & $87.8 \pm 3.0$ & $63.9 \pm 6.7 *, \dagger$ & $47.9 \pm 3.8$ & $43.9 \pm 3.1$ & $46.0 \pm 2.5$ \\
\hline Hepatic TG ( $\mu \mathrm{g} / \mathrm{mg}$ protein) & 5 & $35.5 \pm 2.1$ & $76.6 \pm 15.9^{*}$ & $30.8 \pm 12.4^{\dagger}$ & $38.8 \pm 7.7$ & $158.2 \pm 34.0^{*}$ & $39.2 \pm 18.1^{\dagger}$ \\
\hline Muscle TG ( $\mu \mathrm{g} / \mathrm{mg}$ protein) & 5 & na & na & na & $4.8 \pm 0.5$ & $10.6 \pm 1.4^{*}$ & $5.8 \pm 1.9^{\dagger}$ \\
\hline Fasting blood glucose $(\mathrm{mmol} / \mathrm{l})$ & 5 & $4.5 \pm 0.3$ & $5.4 \pm 0.1$ & $5.1 \pm 0.2$ & $5.1 \pm 0.1$ & $5.5 \pm 0.2$ & $5.0 \pm 0.1$ \\
\hline Fed blood glucose $(\mathrm{mmol} / \mathrm{l})$ & 5 & $8.2 \pm 0.2$ & $9.0 \pm 0.1 *$ & $7.9 \pm 0.3^{\dagger}$ & $8.0 \pm 0.3$ & $9.1 \pm 0.3^{*}$ & $7.7 \pm 0.4^{\dagger}$ \\
\hline
\end{tabular}

Values are expressed as mean \pm SEM

Values are not repeated measures, as the older mice were not the same as the younger ones

Male mice were fasted overnight before blood was drawn and tissues excised

NMR (Bruker Minispec) and ${ }^{1} \mathrm{H}-\mathrm{MRS}$ (Echo Medical Systems) were used to determine fat and lean mass

Nonparametric data were analysed by the Kruskal-Wallis test with Dunn's correction for multiple pairwise comparisons ${ }^{*} p \leq 0.05 \mathrm{vs} C c 1^{+/+},{ }^{\dagger} p \leq 0.05$ vs $C c 1^{-/ \text {-xliver+ }}$

BW, body weight; MRS, magnetic resonance spectroscopy; na, non-applicable; TG, triacylglycerol

(p-CEACAM1 detection in the FASN immunopellet) (Fig. 3j, IP: $\alpha$-FASN; IB: $\alpha$-p-CEACAM1). In $C c 1^{-/}$mice, however, when hyperinsulinaemia develops, insulin receptors are downregulated by $\sim 65 \%$ (Fig. 3i; IB: $\alpha$-p-IR $\beta$ ) $[2,3]$, blunting insulin-initiated IR $\beta$ activation and CEACAM1 phosphorylation and its binding to FASN (Fig. 3j; IP: $\alpha$ FASN; IB: $\alpha$-p-CEACAM1). This halts the negative effect of insulin on FASN activity in refed mice (Fig. 3k). Because FASN levels were induced by hyperinsulinaemia in $\mathrm{CCl}^{-/-}$mice, probably mediated by SREBP-1c activation (ESM Table 2) [22], basal FASN activity was higher in $\mathrm{Ccl}^{-/}$ than in normoinsulinaemic mice and remained uncountered at refeeding in the absence of CEACAM1 (Fig. 3k).

$\mathrm{CCl}^{-/-}$mice exhibited multiple foci of inflammatory cell infiltrates in the hepatic lobules without noticeable ballooning injury of the hepatocyte (Fig. 3b). qPCR analysis revealed elevation in mRNA levels of inflammatory markers such as hepatic tumour necrosis factor- $\alpha(\operatorname{Tnf} \alpha)$ and Cox 2 in $\mathrm{Ccl}^{-/-}$, parallel to an increase in their macrophage population (F4/80 mRNA) (ESM Table 3). The inflammatory response to $\mathrm{Ccl}$ deletion was reversed by liver-specific rescuing of CEACAM1, as demonstrated by the loss of inflammatory infiltrates in the H\&E-stained sections (Fig. 3d) and normalisation of mRNA levels of inflammatory markers in the livers of $\mathrm{Ccl}^{-1-\text { xliver+ }}$ mice (ESM Table 3).

Hepatic CEACAM1 rescuing prevents visceral adiposity and inflammation in $\boldsymbol{C c I}^{-1}$ mice As shown by DeAngelis et al [7], and Gosh et al [11], increased lipid production in the livers of $\mathrm{Ccl}^{-/-}$mice drives redistribution to WAT causing visceral obesity (Table 2). Liver-specific rescuing of CEACAM1 prevented the gain in visceral obesity together with plasma leptin levels (Table 2). This limited lipolysis, indicated by normal plasma NEFA (Table 2), and significantly reduced mRNA levels of inflammatory markers, such as $\operatorname{Tn} f \alpha$, F4/80 and $\mathrm{Il}-1 \beta$, in $\mathrm{Ccl}^{-/- \text {xlivert }}$ mice compared with $\mathrm{Ccl}^{-/-}$ mice (ESM Table 3).

Hypothalamic insulin resistance in $\mathrm{CcI}^{-/-}$mice $\mathrm{Ccl}^{-/-}$mice manifested progressively higher body weight than $\mathrm{CcI}^{+/+}$mice in parallel to hyperphagia and reduced physical activity without significantly affecting energy expenditure (and heat production) at 5-6 months of age when they develop leptin [23] and insulin resistance [7]. To investigate whether insulin resistance contributes to energy imbalance, we subjected mice to indirect calorimetry. As shown in Fig. 4a, b, rescuing hepatic 
Fig. 2 Hyperinsulinaemiceuglycaemic clamp analysis performed on 6-month-old awake overnight-fasted mice.

Measurements under clamp conditions with primed and continuous infusion of insulin are shown for $\mathrm{Ccl}^{+/+}$(white bars),

$\mathrm{Ccl}^{-/}$(black bars) and

$\mathrm{CCl}^{- \text {-xliver+ }}$ (dark grey bars) mice ( $n=8-9 /$ genotype). In (a), (b) and (e), measurements for the basal condition (light grey bars in all genotypes) are also shown.

Values are expressed as mean \pm $\mathrm{SEM} ; *{ }^{*} \leq 0.05$ vs $C c 1^{+/+}$, ${ }^{\dagger} p \leq 0.05 \mathrm{vs} \mathrm{Ccl}{ }^{-/-},{ }^{\ddagger} p \leq 0.05$ vs basal. Gastroc., gastrocnemius; $R_{\mathrm{a}}$, rate of appearance; $R_{\mathrm{d}}$, rate of disposal; WB, whole body
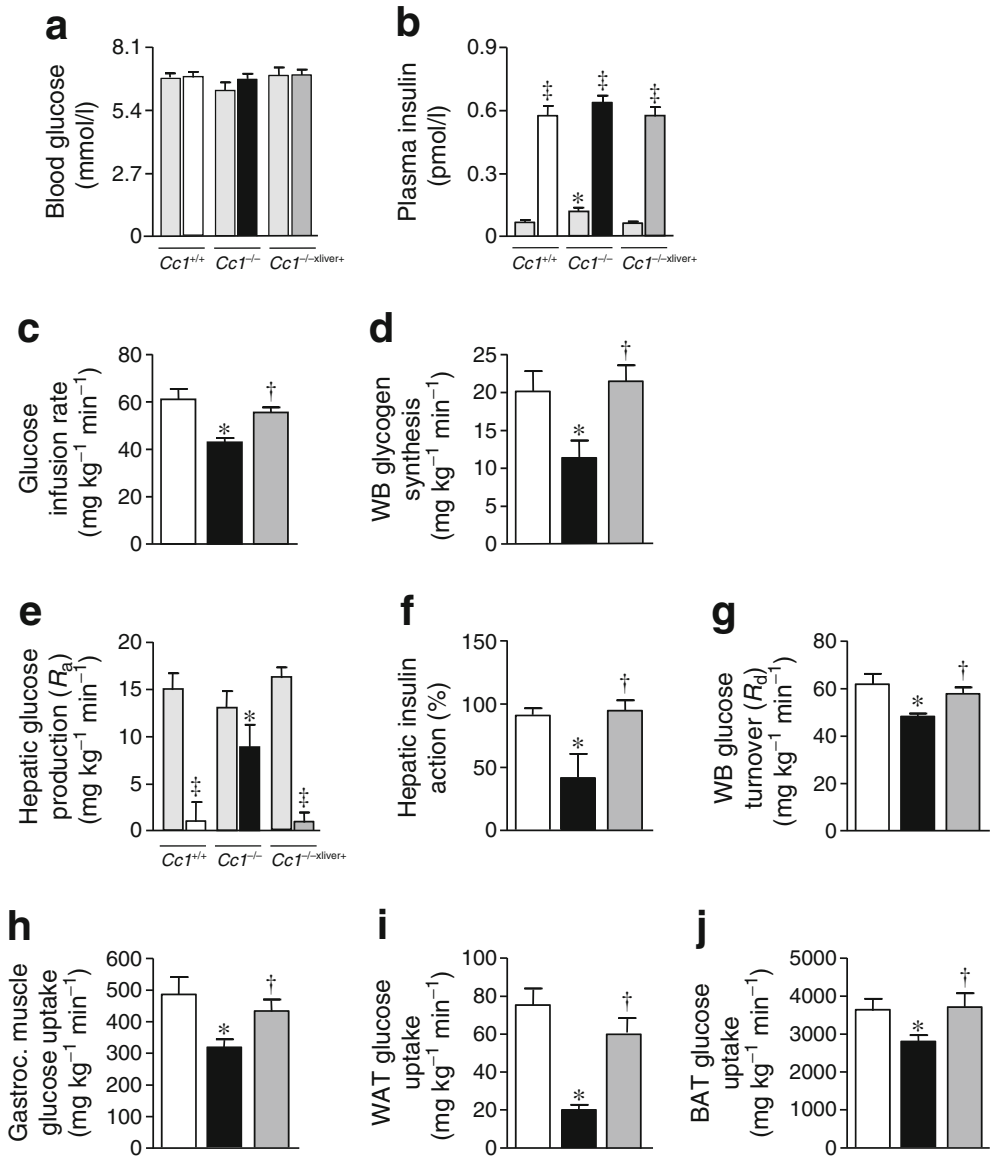

CEACAM1 reversed hyperphagia and physical inactivity in $\mathrm{CCl}^{-/-}$mice, respectively, in particular in the dark cycle when the phenotype is more pronounced [23]. As expected, there was no significant effect on heat production (Fig. 4c). This is supported partly by the reversal of triacylglycerol content in skeletal muscle (Table 2). As expected from the downregulatory effect of hyperinsulinaemia on insulin receptors [2, 3], western blot analysis using IR $\beta$ antibodies revealed lower insulin receptor levels in hypothalamic lysates from $\mathrm{Ccl}^{-/}$than $\mathrm{Ccl}^{+/+}$ and $\mathrm{Ccl}^{-1 \text {-xliver+ }}$ mice (Fig. 5a). Consistently, insulin release during refeeding failed to activate the insulin receptor in the hypothalamus of $\mathrm{CCl}^{-/-}$mice, as it did in $\mathrm{Ccl}^{+/+}$and $\mathrm{Ccl}^{-1-\text { xliver+ }}$ mice (assessed by immunoblotting with $\alpha$-pIR $\beta$ ) (Fig. 5a). Together, this demonstrates that insulin resistance in the hypothalamus contributes to energy imbalance in $\mathrm{Ccl}^{-/-}$mice.

Because hypothalamic FASN activation causes hyperphagia independent of leptin [24-26], we then examined FASN activity in the hypothalamus. As Fig. 5b, c indicate, basal mRNA and protein levels of hypothalamic FASN and its activity were elevated in $\mathrm{CCl}^{-/-}$mice, but not in normoinsulinaemic $\mathrm{CCl}^{- \text {-xliver+ }}$ mice. Western blot analysis (Fig. 5c) shows that, as in the liver, insulin release during refeeding caused CEACAM1 phosphorylation and association with the FASN immunopellet in the normoinsulinaemic $\mathrm{CcI}^{+/+}$mice. This caused reduction in FASN activity in refed relative to fasted wild-type mice (Fig. 5d). Reduction in FASN activity in refed $\mathrm{CCl}^{-/ \text {-xliver+ }}$ mice in response to insulin was due to the phosphorylation and binding of CEACAM2 to FASN, as shown by the identification of a p-CEACAM2 band in the $\alpha$-FASN immunopellet that was not detected when the gel was reimmunoblotted with $\alpha$-CEACAM1 (Fig. $5 \mathrm{c}$ ). Of note, because the cytoplasmic domain of CEACAM2 shares a high homology with that of CEACAM1, in particular at the tyrosine site [5], p-CEACAM1 antibodies also recognise p-CEACAM2 which is highly expressed in the hypothalamus [27]. To investigate whether the increase in hypothalamic FASN activity contributes to hyperphagia, we examined the effect of C75, a FASN inhibitor [28], on daily food intake. As shown in Heinrich et al [23], $\mathrm{Ccl}^{-/-}$mice exhibit a higher daily food intake than $\mathrm{CcI}^{+/+}$ mice (Fig. 5e). Treatment with $\mathrm{C} 75$ for 4 days reversed hyperphagia (Fig. 5e) and elevated body weight (Fig. 5f) in $\mathrm{Ccl}^{-/-}$ mice. The data indicate that the rise in hypothalamic FASN level and activation contributes to elevated food intake and obesity in $\mathrm{Ccl}^{-/-}$mice.

Role of lipolysis in insulin resistance in $\boldsymbol{C c I}^{-/-}$mice Next, we examined the effect of preventing lipolysis with nicotinic 
a

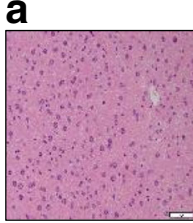

b

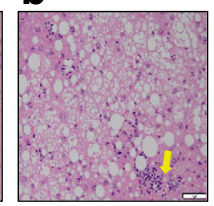

e
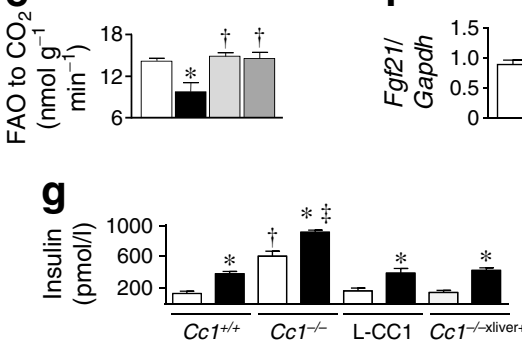

h

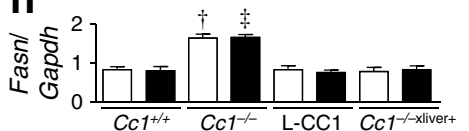

i

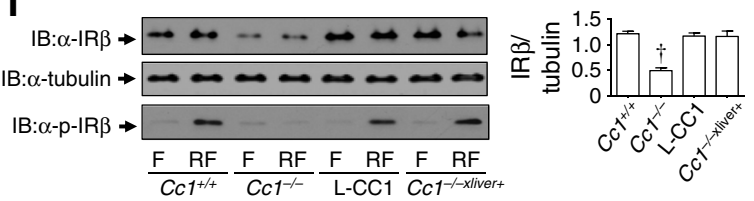

j

IP: $\alpha$-FASN

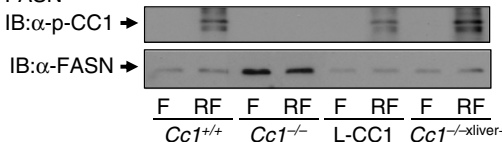

k

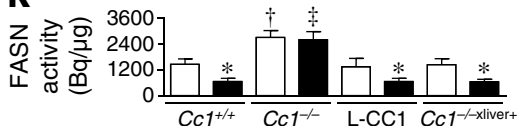

acid on insulin signalling and insulin intolerance. As shown in Fig. 6a, nicotinic acid reduced plasma NEFA by $\sim 50 \%$ in all groups, normalising that of $C \mathrm{Cl} l^{-/-}$mice to the level of untreated $\mathrm{Cll}^{+/ 4}$ mice. However, it failed to reverse impairment of insulin clearance (C-peptide/insulin molar ratio) (Fig. 6d) and hyperinsulinaemia (Fig. 6b). It also failed to reverse insulin resistance, as demonstrated by persistent insulin intolerance in $\mathrm{CcI}^{-/-}$mice (Fig. 6e, f) and blunted insulin receptor phosphorylation in the liver, hypothalamus and WAT of $\mathrm{Ccl}^{-1-}$ mice in response to insulin release during refeeding (ESM Fig. 2). As expected [17], nicotinic acid increased macrophage recruitment to the WAT of all groups (ESM Table 4) to blunt insulin action in the WAT of refed control mice (ESM Fig. 2). Together, this demonstrates that lipolysis is the consequence rather than the cause of systemic insulin resistance in $\mathrm{CCl}^{-/-}$ mice.
Fig. 3 Lipid metabolism in the liver. (a-d) H\&E staining in the liver of 8month-old $\mathrm{Ccl}^{+/+}$(a), $\mathrm{Ccl}^{-/-}$(b), L-CC1 (c) and $\mathrm{Ccl}^{-/ \text {-xliver+ }}$ (d) mice ( $n=5 /$ genotype). Yellow arrow points to foci of inflammatory cell infiltrates. (e) Hepatic FAO (palmitate) in fasted $\mathrm{Ccl}^{+/+}$(white bars), $\mathrm{Ccl}^{-1-}$ (black bars), L-CC1 (light grey bars) and $\mathrm{Ccl}^{-/-x l i v e r+}$ (dark grey bars) mice ( $n=5 /$ genotype). Assays were performed in triplicate. (f) mRNA analysis of $F g f 21$ (performed in triplicate) in the livers of $\mathrm{CcI}^{+/+}$ (white bars), $\mathrm{Ccl}^{-1-}$ (black bars), L-CC1 (light grey bars) and $\mathrm{Ccl}^{-/- \text {xliver+ }}$ (dark grey bars) mice $(n=5 ; 6$ months of age). Values are expressed as mean \pm SEM; $* p \leq 0.05$ vs $C c l^{+/+}$and ${ }^{\dagger} p \leq 0.05$ vs $C c 1^{-/-} ;(\mathbf{g}-\mathbf{j})$ Mice ( 2 months of age) were fasted overnight (white bars or ' $F$ ') and refed for $7 \mathrm{~h}$ (black bars or 'RF'). (g, h) Analysis of plasma insulin levels $(n=6$ per genotype per feeding state) (g) and Fasn mRNA expression relative to Gapdh ( $n=5$ per genotype per feeding state; performed in triplicate) (h). Values are expressed as mean \pm SEM. (i) Western blot analysis of liver lysates was performed to assess insulin receptor protein level $(\alpha$-IR $\beta)$ and phosphorylation $(\alpha$-p-IR $\beta)$. Immunoblotting with $\alpha$-tubulin was carried out for normalisation. Quantification of IR $\beta$ to tubulin was measured by densitometry in fasting samples. (j) Some aliquots were subjected to immunoprecipitation with $\alpha$-FASN followed by immunoblotting with $\alpha$ p-CEACAM1 antibody $(\alpha-\mathrm{p}-\mathrm{CC} 1)$. Gels represent two separate experiments performed on different mice per genotype per feeding state. (k) FASN activity was measured in triplicate by $\left[{ }^{14} \mathrm{C}\right]$ malonyl-CoA incorporation ( $n=5$ per genotype per feeding state). Values are expressed as mean \pm SEM. For $(\mathbf{g}-\mathbf{i})$ and $(\mathbf{k}), * p<0.05$ refed vs fasted per genotype, ${ }^{\dagger} p \leq 0.05 \mathrm{Ccl}^{-/}$vs other genotypes at fasting, ${ }^{\dagger} p \leq 0.05 \mathrm{vs}$ other genotypes at refeeding. IB, immunoblotting; IP, immunoprecipitation

\section{Discussion}

Consistent with the upregulatory role of CEACAM1 in receptor-mediated insulin internalisation and degradation in the hepatocyte, $\mathrm{Ccl}^{-/}$mice develop impaired insulin clearance with chronic hyperinsulinaemia, in addition to insulin resistance and hepatic steatosis [7, 12]. As recovery of insulin sensitivity and lipid homeostasis, in addition to visceral obesity are observed in $\mathrm{CCl}^{- \text {-xliver+ }}$ mice, a key role can be assigned to hepatic CEACAM1-dependent pathways in regulating insulin and lipid homeostasis and systemic insulin action.

Downregulation of hepatic and hypothalamic insulin receptors in $\mathrm{CCl}^{-/}$mice and subsequent manifestation of cellular insulin resistance could result from a common pathway such as systemic hyperinsulinaemia that begins at 2 months of age. Insulin resistance caused by hyperinsulinaemia has been shown in cells [2, 29], in mouse livers [30] and in humans with benign insulinoma [31]. As expected [22], hyperinsulinaemia induced SREBP1c-mediated transcription of FASN in $\mathrm{CCl}^{-/-}$mice and, subsequently, its activation, since it simultaneously relieves the hepatocyte from the acute negative effect of insulin on FASN activity, owing to compromised insulin pulsatility [4] and the absence of the counterregulatory effect of CEACAM1 [13]. This increases de novo lipogenesis and substrate redistribution to the white adipose depot to cause visceral obesity followed by lipolysis and systemic insulin resistance. While this may cause a compensatory increase in insulin secretion that could, in turn, contribute to 

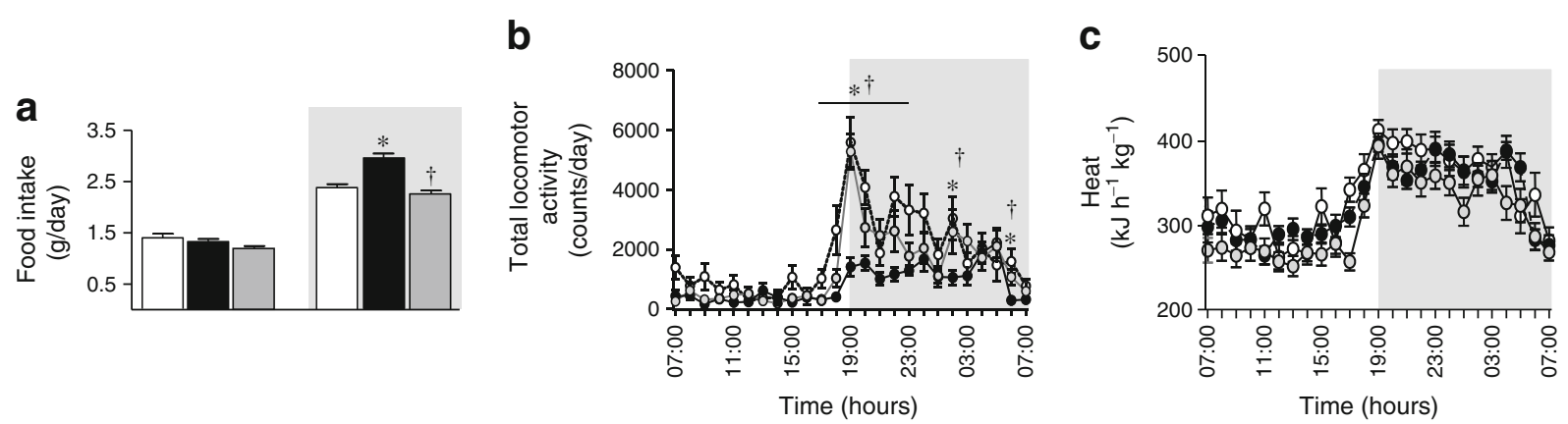

Fig. 4 Energy balance. Six-month-old $\mathrm{Ccl}^{+/+}$(white), $\mathrm{Ccl}^{-/-}$(black) and $\mathrm{CCl}^{-1-\text { xliver }}$ (grey) mice were individually caged $(n=4 /$ genotype) and analysed by indirect calorimetry (CLAMS system) for 5 days to measure (a) daily food intake, (b) total locomotor activity (counts/day) and (c) heat production $\left(\mathrm{kJ} \mathrm{h}^{-1} \mathrm{~kg}^{-1}\right)$ every $20 \mathrm{~min}$ at a flow rate of $0.5 \mathrm{l} / \mathrm{min}$ for $24 \mathrm{~h}$.

the chronic hyperinsulinaemic state [32], persistence of hyperinsulinaemia and insulin resistance in $\mathrm{Ccl}^{-/-}$mice after

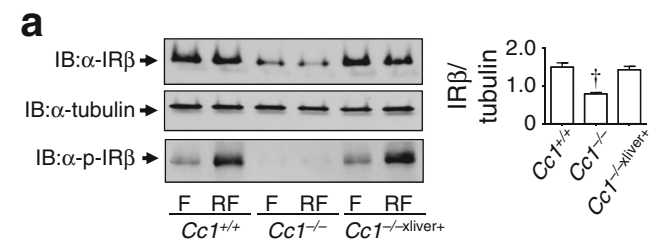

b
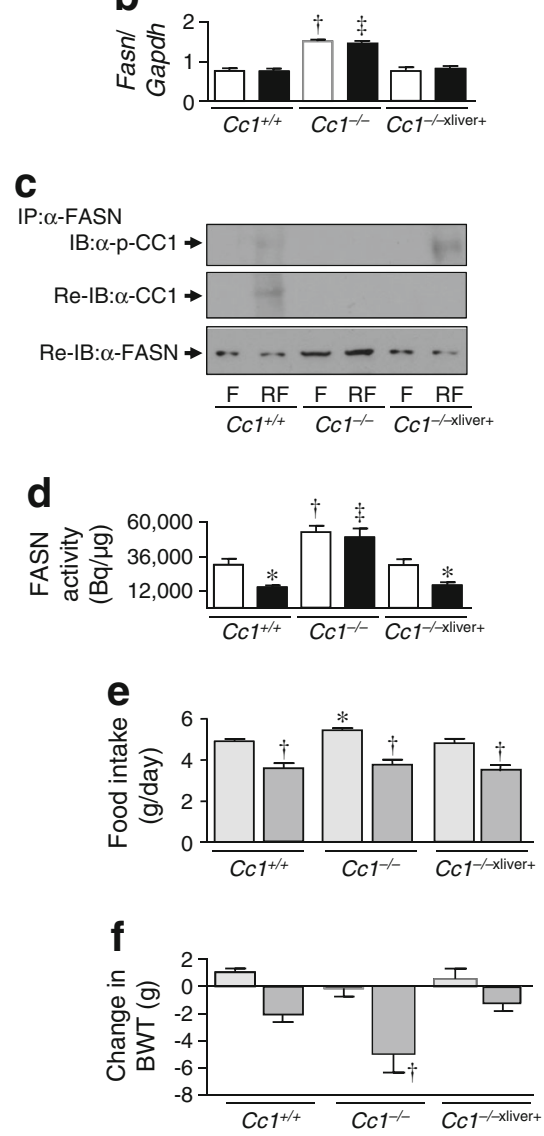

Values are expressed as mean $\pm \mathrm{SEM}$ of each time interval in the last 3 days in the light (07:00 hours to 19:00 hours) and dark (shaded; 19:00 hours to 07:00 hours) cycle. ${ }^{*} p \leq 0.05 \mathrm{Ccl}^{-/-}$vs $C c 1^{+/+},{ }^{\dagger} p \leq 0.05$ $\mathrm{Ccl}^{-/ \text {-xlivert }}$ vs $\mathrm{CCl}^{-/}$

blocking lipolysis with nicotinic acid demonstrates that these metabolic abnormalities arise independent of visceral obesity and that they are the cause rather than the consequence of lipolysis.

Null deletion of $C c 1$ induces chronic hyperinsulinaemia and hepatic de novo lipogenesis, which, combined with reduced fatty acid $\beta$-oxidation and increase NEFA uptake, leads to hepatic steatosis [11]. It also promotes visceral obesity and release of proinflammatory adipokines that contribute to systemic insulin resistance [33]. Together with increased recruitment of macrophages, infiltrating adipokines modulate the inflammatory milieu of the liver parenchyma to cause

Fig. 5 Regulation of hypothalamic FASN activity. Hypothalami were extracted from the same 2-month-old mice used in Fig. $3 \mathrm{~g}-\mathrm{k}$ after being fasted ('F' or white bars) or refed for $7 \mathrm{~h}$ ('RF' or black bars). (a) Lysates were analysed by immunoblotting with $\alpha-\operatorname{IR} \beta$ or $\alpha$-p-IR $\beta$, using $\alpha$ tubulin for normalisation. (b) mRNA analysis of Fasn relative to Gapdh ( $n=5$ per genotype per feeding state) was performed in triplicate. Values are expressed as mean $\pm \mathrm{SEM} ;{ }^{\dagger} p \leq 0.05 \mathrm{Ccl}^{-/-}$vs other genotypes at fasting, ${ }^{\star} p \leq 0.05$ vs other genotypes at refeeding. (c) As in Fig. 3j, some aliquots were subjected to immunoprecipitation with $\alpha$-FASN followed by immunoblotting with the $\alpha$-p-CEACAM1 antibody $(\alpha-p-C C 1)$ that also recognises CEACAM2 (a related protein with a cytoplasmic tail that shares a very high homology with that of CEACAM1). Proteins were re-immunoblotted with $\alpha$-CEACAM1 antibody $(\alpha-\mathrm{CC} 1)$ for normalisation and with $\alpha$-FASN antibody to account for the amount of immunoprecipitated FASN. Gels represent two separate experiments performed on different mice per genotype per feeding state. (d) FASN activity was measured in triplicate by $\left[{ }^{14} \mathrm{C}\right]$ malonyl-CoA incorporation ( $n=5$ per genotype per feeding state). Values are expressed as mean $\pm \mathrm{SEM} ; * p<0.05$ refed vs fasted per genotype, ${ }^{\dagger} p \leq 0.05 \mathrm{Ccl}^{-/}$vs other genotypes at fasting, ${ }^{\star} p \leq 0.05 \mathrm{vs}$ other genotypes at refeeding; (e) Daily food intake ( $n=6$ per genotype per treatment; 6-month-old mice) and (f) body weight (BWT) $(n=6$ per genotype per treatment; 6-month-old) were assessed over a period of 4 days in mice receiving an i.p. injection of vehicle (Veh, light grey bars) or C75 (dark grey bars). Data are presented as the difference between day 4 and day 0 of treatment. Values are expressed as mean \pm SEM. For (e) and (f), ${ }^{*} p<0.05$ vs vehicle-treated $C c 1^{+/+},{ }^{\dagger} p<0.05$ vs vehicle-treated within genotype. IB, immunoblotting; IP, immunoprecipitation 

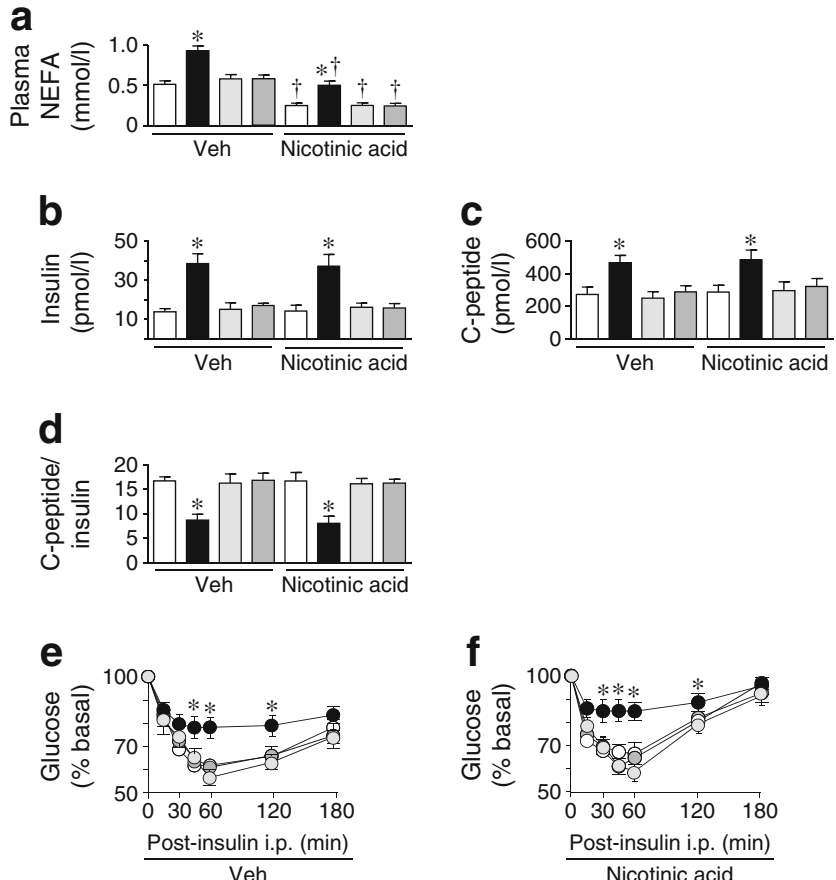

Fig. 6 Metabolic effect of nicotinic acid on insulin clearance and tolerance. (a) Six-month-old mice were treated with vehicle (Veh) or two injections of nicotinic acid ( $n=6$ per genotype per treatment) before being fasted until 11:00 hours and plasma NEFA was assayed. (b-d) Insulin and C-peptide were assayed and the molar ratio measured as a marker of insulin clearance. (e, f) Insulin tolerance was also assessed in mice treated with Veh (e) or nicotinic acid (f). $\mathrm{Ccl}^{+/+}$, white; $\mathrm{Ccl}^{-/}$, black; L-CC1, light grey; $C c 1^{-1-x l i v e r+}$, dark grey. Values are expressed as mean \pm SEM. For $(\mathbf{a}-\mathbf{d}),{ }^{*} p \leq 0.05$ vs $C c 1^{+++},{ }^{\dagger} p \leq 0.05 C c 1^{-1-x \text { liver+ }}$ vs $\mathrm{Ccl}^{-/-}$; for $(\mathbf{e}-\mathbf{f}),{ }^{*} p \leq 0.05, \mathrm{Ccl}^{+/+}$vs other mice

steatohepatitis. The critical regulatory role of the loss of hepatic CEACAM1-dependent pathways in these metabolic abnormalities is further demonstrated by the curbed visceral adiposity with associated lipolysis and recruitment of proinflammatory cytokines in $\mathrm{CcI}^{- \text {-xliver+ }}$ mice. This protective metabolic effect of hepatic CEACAM1 is consistent with the prevention of diet-induced insulin resistance and metabolic abnormalities by transgenic overexpression of CEACAM1 in the liver of C57BL/6 mice $[15,34]$ and by their reversal with liver-specific adenoviral-mediated delivery [17].

$\mathrm{Ccl}^{-/-}$mice also develop obesity and higher total fat mass by 6 months of age, owing to hyperphagia and low physical activity [23]. Reversal of this energy imbalance in parallel to restoring physiological insulin levels and hypothalamic insulin signalling in $\mathrm{CCl}^{-/ \text {-xlivert }}$ mice points to the contribution of hypothalamic insulin resistance in the energy imbalance of global $\mathrm{CCl}^{-/-}$mice, in support of the well-documented role of insulin in regulating food intake and physical activity [35-37], as well as insulin response in liver [38].

Mechanistically, hyperinsulinaemia causes obesity [39], in part by inducing leptin resistance and hyperphagia [40]. Thus, normalisation of food intake can also result from reduced visceral obesity by liver-specific recovery of CEACAM1 expression. Hyperinsulinaemia also drives FASN transcription [22] and, subsequently, its activity. Reversal of hyperphagia and obesity in $\mathrm{Ob} / \mathrm{Ob}$ mice by $\mathrm{C} 75$ demonstrates that inactivating hypothalamic FASN reduces food intake independently of leptin [24-26]. Consistently, hypothalamic FASN deletion suppresses food intake [41]. Herein we show for the first time that upon its phosphorylation by the insulin receptor, hypothalamic CEACAM1 binds to FASN to reduce its activity in response to the acute rise of insulin in normoinsulinaemic mice, as it does in liver [13]. In the hyperinsulinaemic $\mathrm{CCl}^{-/-}$mice, basal FASN activity is elevated as a result of the rise in its insulin-induced SREBP1c-mediated transcription and level. In the absence of the counterregulatory effect of CEACAM1, this maintains high FASN activity to contribute to hyperphagia, as demonstrated by the reversal effect of $\mathrm{C} 75 \mathrm{FASN}$ inhibitor on food intake in $\mathrm{Ccl}^{-/}$ mice. Liver-specific rescuing of CEACAM1 restored normoinsulinaemia and the negative effect of insulin on FASN activity by promoting phosphorylation and binding of CEACAM2 - a relative of CEACAM1 that shares a very strong amino acid homology in its cytoplasmic domain. This emphasises the dependence of the negative effect of insulin on FASN activity on the prior insulinaemic state and, subsequently, on CEACAM phosphorylation [13]. It also provides a potential mechanism linking normalisation of insulin homeostasis and action to reduced food intake in $\mathrm{Cc}^{-/- \text {xliver+ }}{ }^{\text {mice. }}$

In summary, the current study demonstrates reversal of insulin resistance, steatohepatitis, obesity and other metabolic abnormalities in $\mathrm{CCl}^{-/-}$mice by liver-specific rescuing of CEACAM1. Given that this normalisation occurs despite persistent loss of CEACAM1 from extrahepatic tissues, the data emphasise a key role for hepatic CEACAM1-dependent pathways in regulating insulin metabolism and action in a coordinated manner with lipid homeostasis and visceral obesity. Together with the reversal effect of adenoviral-mediated hepatic CEACAM1 delivery on diet-induced insulin resistance, hepatic steatosis and visceral obesity, this identifies induction of liver CEACAM1 as a potential therapeutic option against metabolic abnormalities. The clinical implications of these findings are emphasised by a marked decrease in hepatic CEACAM1 levels in obese individuals with fatty liver disease and insulin resistance $[42,43]$.

Acknowledgements The authors thank N. Beauchemin (McGill University, Montréal, QC, Canada) for her gift of the custom-made rabbit Ab2456 and Ab3759 polyclonal antibodies. We also thank J. Kalisz and M. Kopfman at the Najjar Laboratory at the University of Toledo College of Medicine for their technical assistance in the generation and maintenance of mice, and in carrying out routine RNA analyses.

Data availability Data are available on request from the corresponding senior author. 
Funding This work was supported by grants from the National Institutes of Health (R01-DK054254, R01-DK083850 and R01HL112248 to SMN, and U24-DK093000 to the National Mouse Metabolic Phenotyping Center at UMass). It was also supported by fellowships from the American Heart Association (14POST20480294) to LR and the Middle-East Diabetes Research Center to HEG and SSG. The studies were also partially supported by the John J. Kopchick PhD OHF Eminent Research Chair Fund.

Duality of interest The authors declare that there is no duality of interest associated with this manuscript.

Contribution statement LR, HTM, HEG, SSG, TAB, H-LN and SD researched data and drafted parts of the manuscript. HTM and HEG revised the manuscript extensively. JKK carried out the hyperinsulinaemiceuglycaemic clamp experiment, and analysed and discussed data. GYD and GH carried out the statistical analysis. GYD, GH and JKK critically reviewed the manuscript. SMN was responsible for study design, conceptualisation, data analysis and interpretation, and reviewing the manuscript. All authors have given final approval of this version to be published. SMN had full access to all the data of the study and takes responsibility for the integrity and accuracy of data analysis and the decision to submit and publish the manuscript

\section{References}

1. Petersen KF, Dufour S, Savage DB et al (2007) The role of skeletal muscle insulin resistance in the pathogenesis of the metabolic syndrome. Proc Natl Acad Sci U S A 104:12587-12594

2. Cook JR, Langlet F, Kido Y, Accili D (2015) Pathogenesis of selective insulin resistance in isolated hepatocytes. J Biol Chem 290: 13972-13980

3. Shanik MH, Xu Y, Skrha J, Dankner R, Zick Y, Roth J (2008) Insulin resistance and hyperinsulinemia: is hyperinsulinemia the cart or the horse? Diabetes Care 31(Suppl 2):S262-S268

4. Matveyenko AV, Liuwantara D, Gurlo T et al (2012) Pulsatile portal vein insulin delivery enhances hepatic insulin action and signaling. Diabetes 61:2269-2279

5. Han E, Phan D, Lo P et al (2001) Differences in tissue-specific and embryonic expression of mouse Ceacam1 and Ceacam2 genes. Biochem J 355:417-423

6. Poy MN, Yang Y, Rezaei K et al (2002) CEACAM1 regulates insulin clearance in liver. Nat Genet 30:270-276

7. DeAngelis AM, Heinrich G, Dai T et al (2008) Carcinoembryonic antigen-related cell adhesion molecule 1: a link between insulin and lipid metabolism. Diabetes 57:2296-2303

8. Heinrich G, Ghadieh HE, Ghanem SS et al (2017) Loss of hepatic CEACAM1: a unifying mechanism linking insulin resistance to obesity and non-alcoholic fatty liver disease. Front Endocrinol (Lausanne) 8:8

9. Najjar SM, Philippe N, Suzuki Y et al (1995) Insulin-stimulated phosphorylation of recombinant pp120/HA4, an endogenous substrate of the insulin receptor tyrosine kinase. Biochemistry 34: 9341-9349

10. Choice CV, Howard MJ, Poy MN, Hankin MH, Najjar SM (1998) Insulin stimulates pp120 endocytosis in cells co-expressing insulin receptors. J Biol Chem 273:22194-22200

11. Ghosh S, Kaw M, Patel PR et al (2010) Mice with null mutation of Ceacam I develop nonalcoholic steatohepatitis. Hepat Med: Res Evidence 2010:69-78

12. Xu E, Dubois MJ, Leung $\mathrm{N}$ et al (2009) Targeted disruption of carcinoembryonic antigen-related cell adhesion molecule 1 promotes diet-induced hepatic steatosis and insulin resistance. Endocrinology 150:3503-3512

13. Najjar SM, Yang Y, Fernstrom MA et al (2005) Insulin acutely decreases hepatic fatty acid synthase activity. Cell Metab 2:43-53

14. Dai T, Abou-Rjaily GA, Al-Share QY et al (2004) Interaction between altered insulin and lipid metabolism in CEACAM1-inactive transgenic mice. J Biol Chem 279:45155-45161

15. Al-Share QY, DeAngelis AM, Lester SG et al (2015) Forced hepatic overexpression of CEACAM1 Curtails diet-induced insulin resistance. Diabetes 64:2780-2790

16. Ramakrishnan SK, Russo L, Ghanem SS et al (2016) Fenofibrate decreases insulin clearance and insulin secretion to maintain insulin sensitivity. J Biol Chem 291:23915-23924

17. Russo L, Ghadieh HE, Ghanem SS et al (2016) Role for hepatic CEACAM1 in regulating fatty acid metabolism along the adipocyte-hepatocyte axis. J Lipid Res 57:2163-2175

18. Huang J, Ledford KJ, Pitkin WB, Russo L, Najjar SM, Siragy HM (2013) Targeted deletion of murine CEACAM 1 activates PI3KAkt signaling and contributes to the expression of (Pro)renin receptor via CREB family and NF-kappaB transcription factors. Hypertension 62:317-323

19. Najjar SM, Ledford KJ, Abdallah SL et al (2013) Ceacam1 deletion causes vascular alterations in large vessels. Am J Physiol Endocrinol Metab 305:E519-E529

20. Houde C, Roy S, Leung N, Nicholson DW, Beauchemin N (2003) The cell adhesion molecule CEACAM1-L is a substrate of caspase3-mediated cleavage in apoptotic mouse intestinal cells. J Biol Chem 278:16929-16935

21. Pereira S, Park E, Mori Y et al (2014) FFA-induced hepatic insulin resistance in vivo is mediated by $\mathrm{PKC} \delta$, NADPH oxidase, and oxidative stress. Am J Physiol Endocrinol Metab 307:E34-E46

22. Wong RH, Chang I, Hudak CS, Hyun S, Kwan HY, Sul HS (2009) A role of DNA-PK for the metabolic gene regulation in response to insulin. Cell 136:1056-1072

23. Heinrich G, Russo L, Castaneda TR et al (2016) Leptin resistance contributes to obesity in mice with null mutation of carcinoembryonic antigen-related cell adhesion molecule 1. J Biol Chem 291:11124-11132

24. Shimokawa T, Kumar MV, Lane MD (2002) Effect of a fatty acid synthase inhibitor on food intake and expression of hypothalamic neuropeptides. Proc Natl Acad Sci U S A 99:66-71

25. Mobbs CV, Makimura H (2002) Block the FAS, lose the fat. Nat Med 8:335-336

26. Kumar MV, Shimokawa T, Nagy TR, Lane MD (2002) Differential effects of a centrally acting fatty acid synthase inhibitor in lean and obese mice. Proc Natl Acad Sci U S A 99:1921-1925

27. Heinrich G, Ghosh S, Deangelis AM et al (2010) Carcinoembryonic antigen-related cell adhesion molecule 2 controls energy balance and peripheral insulin action in mice. Gastroenterology 139:644-652

28. Loftus TM, Jaworsky DE, Frehywot GL et al (2000) Reduced food intake and body weight in mice treated with fatty acid synthase inhibitors. Science 288:2379-2381

29. Gonzalez E, Flier E, Molle D, Accili D, McGraw TE (2011) Hyperinsulinemia leads to uncoupled insulin regulation of the GLUT4 glucose transporter and the FoxO1 transcription factor. Proc Natl Acad Sci U S A 108:10162-10167

30. Yang X, Mei S, Gu H et al (2014) Exposure to excess insulin (glargine) induces type 2 diabetes mellitus in mice fed on a chow diet. J Endocrinol 221:469-480

31. Battezzati A, Terruzzi I, Perseghin G et al (1995) Defective insulin action on protein and glucose metabolism during chronic hyperinsulinemia in subjects with benign insulinoma. Diabetes 44:837-844

32. Broussard JL, Kolka CM, Castro AV et al (2015) Elevated nocturnal NEFA are an early signal for hyperinsulinaemic compensation 
during diet-induced insulin resistance in dogs. Diabetologia 58: 2663-2670

33. Najjar SM, Russo L (2014) CEACAM1 loss links inflammation to insulin resistance in obesity and non-alcoholic steatohepatitis (NASH). Semin Immunopathol 36:55-71

34. Lester SG, Russo L, Ghanem SS et al (2015) Hepatic CEACAM1 over-expression protects against diet-induced fibrosis and inflammation in white adipose tissue. Front Endocrinol (Lausanne) 6:116-122

35. Obici S, Rossetti L (2003) Minireview: nutrient sensing and the regulation of insulin action and energy balance. Endocrinology 144:5172-5178

36. Woods SC, Lotter EC, McKay LD, Porte D Jr (1979) Chronic intracerebroventricular infusion of insulin reduces food intake and body weight of baboons. Nature 282:503-505

37. Plum L, Belgardt BF, Bruning JC (2006) Central insulin action in energy and glucose homeostasis. J Clin Invest 116:1761-1766

38. Obici S, Zhang BB, Karkanias G, Rossetti L (2002) Hypothalamic insulin signaling is required for inhibition of glucose production. Nat Med 8:1376-1382
39. D'Souza AM, Johnson JD, Clee SM, Kieffer TJ (2016) Suppressing hyperinsulinemia prevents obesity but causes rapid onset of diabetes in leptin-deficient Lepob/ob mice. Mol Metab 5:1103-1112

40. Kellerer M, Lammers R, Fritsche A et al (2001) Insulin inhibits leptin receptor signalling in HEK293 cells at the level of janus kinase-2: a potential mechanism for hyperinsulinaemia-associated leptin resistance. Diabetologia 44:1125-1132

41. Chakravarthy MV, Zhu Y, Yin L et al (2009) Inactivation of hypothalamic FAS protects mice from diet-induced obesity and inflammation. J Lipid Res 50:630-640

42. Lee $\mathrm{W}$ (2011) The CEACAM1 expression is decreased in the liver of severely obese patients with or without diabetes. Diagn Pathol 6: 40

43. Heinrich G, Muturi HT, Rezaei K et al (2017) Reduced hepatic carcinoembryonic antigen-related cell adhesion molecule 1 level in obesity. Front Endocrinol (Lausanne) 8:54 